\title{
The Relationship between Cortical Magnification Factor and Population Receptive Field Size in Human Visual Cortex: Constancies in Cortical Architecture
}

\author{
Ben M. Harvey and Serge 0. Dumoulin \\ Helmholtz Institute, Experimental Psychology, Utrecht University, 3584 CS Utrecht, The Netherlands
}

Receptive field (RF) sizes and cortical magnification factor (CMF) are fundamental organization properties of the visual cortex. At increasing visual eccentricity, RF sizes increase and CMF decreases. A relationship between RF size and CMF suggests constancies in cortical architecture, as their product, the cortical representation of an RF (point image), may be constant. Previous animal neurophysiology studies of this question yield conflicting results. Here, we use fMRI to determine the relationship between the population RF (pRF) and CMF in humans. In average and individual data, the product of CMF and pRF size, the population point image, is near constant, decreasing slightly with eccentricity in V1. Interhemisphere and subject variations in CMF, pRF size, and V1 surface area are correlated, and the population point image varies less than these properties. These results suggest a V1 cortical processing architecture of approximately constant size between humans. Up the visual hierarchy, to V2, V3, hV4, and L01, the population point image decreases with eccentricity, and both the absolute values and rate of change increase. PRF sizes increase between visual areas and with eccentricity, but when expressed in V1 cortical surface area (i.e., corticocortical pRFs), they are constant across eccentricity in V2/V3. Thus, V2/V3, and to some degree $\mathrm{hV} 4$, sample from a constant extent of $\mathrm{V} 1$. This may explain population point image changes in later areas. Consequently, the constant factor determining PRF size may not be the relationship to the local CMF, but rather pRF sizes and CMFs in visual areas from which the pRF samples.

\section{Introduction}

Cortical magnification factor (CMF) and receptive field (RF) size are fundamental properties of visual cortex. The RF is a property of individual neurons, describing the visual field region where visual stimulation elicits a response. CMF is a property of neuronal organization. In early visual cortex, neurons and their RFs are organized into visual field maps (Wandell et al., 2007). CMF indicates that more neurons process the central visual field than peripheral parts. CMF is the cortical surface distance between two points representing visual field positions $1^{\circ}$ apart (Daniel and Whitteridge, 1961).

Both CMF and RF size vary systematically. In virtually all visual field maps, as distance from the visual field center (eccentricity) increases, CMF decreases and RF sizes increase (Hubel and Wiesel, 1962, 1974; Dow et al., 1981; Van Essen et al., 1984). Both properties also change between visual areas. Initial reports in cat (Albus, 1975) and Macaca mulatta (Hubel and Wiesel, 1974) reported a parallel relationship between RF

\footnotetext{
Received May 24, 2011; revised July 19, 2011; accepted July 28, 2011.

Author contributions: B.M.H. and S.O.D. designed research; B.M.H. and S.O.D. performed research; B.M.H. and S.O.D. analyzed data; B.M.H. and S.O.D. wrote the paper.

This work was supported by a Netherlands Organisation for Scientific Research Vidi Grant 452-08-008 (S.0.D.). We thank Dr. I. T. C. Hooge for his help with the eye position control experiments.

Correspondence should be addressed to either Ben M. Harvey or Serge 0. Dumoulin, Experimental Psychology, Utrecht University, Heidelberglaan 2, 3584 CS Utrecht, The Netherlands. E-mail: b.m.harvey@uu.nl or s.o.dumoulin@uu.nl.

DOI:10.1523/JNEUROSCI.2572-11.2011

Copyright $\odot 2011$ the authors $\quad 0270-6474 / 11 / 3113604-09 \$ 15.00 / 0$
}

size and CMF in V1, suggesting that their product, point image size, is approximately constant. The point image is the cortical representation of a given $\mathrm{RF}$, equivalent to cortical surface area activated by a point in visual space. Constant point image suggests a constantly sized processing unit and constancies in cortical architecture.

Dow et al. (1981) challenged these conclusions, extending measurements into the foveal representation. Here, CMF increases without proportional decreases of RF size. McIlwain (1986) interpreted these results as a constant increase of point image in log space toward the fovea. This was supported by Van Essen et al. (1984) in Macaca fascicularis, although they suggested that $>5.5^{\circ}$ eccentricity point image size increases again. These reports are difficult to reconcile. They differ along several experimental dimensions. First, the species differ. Second, some use single-unit and others multiunit recordings. Single-unit measurements confound RF position with RF position scatter. Multiunit measurements [including the population RF ( $\mathrm{pRF}$ )] compensate for scatter by measuring aggregate RF size (McIlwain, 1986), although these aggregate RF sizes combine scatter extent with RF size. Last, measurements were performed in very few hemispheres, generally with different data coming from different hemispheres, and individual variability may account for some differences (Van Essen et al., 1984).

Motter (2009) suggested an alternative view of RF size. Motter found that RFs in macaque V4 become constantly sized across eccentricity when expressed on the visual field map in V1. This suggests a constant relationship of RF size to the CMF of the area 
from which it is sampled (i.e., a constant extent of sampling of $\mathrm{V} 1$ by $\mathrm{V} 4$ ).

Here, we examine this question in humans using functional magnetic resonance imaging (fMRI). Using fMRI, the CMF (Sereno et al., 1995; DeYoe et al., 1996; Dougherty et al., 2003; Wandell et al., 2007) and aggregate RF within an fMRI recording site ( $\mathrm{pRF}$ ) can be routinely determined (Dumoulin and Wandell, 2008; Kay et al., 2008; Amano et al., 2009; Winawer et al., 2010). Both properties vary significantly between hemispheres, and are comparable with neurophysiological results. fMRI provides several advantages: it measures $\mathrm{pRF}, \mathrm{CMF}$, and point image within each recoding site; it distributes recordings homogeneously across visual cortex; and it allows characterization of individual human subjects. We describe a close relationship between pRF size, CMF, and visual area size within and between hemispheres. We propose that the constant factor determining pRF size is the relationship to $\mathrm{pRF}$ sizes and CMFs in visual areas from which the pRF samples, rather than the local CMF.

\section{Materials and Methods}

Subjects. Eleven healthy subjects participated in this study (age range, 23-47; three females). All subjects have normal or corrected-to-normal visual acuity. All experimental procedures were cleared by the ethics committee of University Medical Center Utrecht.

MRI acquisition. Functional and anatomical MRI data were acquired on a Philips Achieva 3T scanner (Philips Medical Systems) with a Quasar Dual gradient set. T1-weighted anatomical MRI data were acquired at a resolution of $0.75 \times 0.75 \times 0.8 \mathrm{~mm}$. Repetition time (TR) was $10.029 \mathrm{~ms}$, echo time (TE) was $4.6 \mathrm{~ms}$, and flip angle was $8^{\circ}$. Functional $\mathrm{T} 2^{\star}$ weighted 2D echo-planar images were acquired at an isotropic resolution of $2.5 \mathrm{~mm}$, with 24 slices. TR was $1500 \mathrm{~ms}$, TE was $30 \mathrm{~ms}$, and flip angle was $70^{\circ}$. Functional scans were each 248 time frames ( $372 \mathrm{~s}$ ) in duration, of which the first eight time frames (12 s) were discarded. Between 7 and 10 repeated scans were acquired within the same session for each subject.

Stimulus presentation setup. Visual stimuli were presented by backprojection onto a $101 \times 76 \mathrm{~cm}$ screen. This was viewed through a mirror attached to the MRI coil. The distance from the subject's eyes (in the scanner) to the display screen, via the mirror, was $348 \mathrm{~cm}$. Display resolution was $600 \times 800$ pixels. Stimuli were constrained to circular area filling the vertical dimension of the screen, with any area outside this circle remaining at constant mean luminance. From the subject's point of view, this stimulus circle had a radius of $6.25^{\circ}$ of visual angle.

Visual stimuli. Visual stimuli were generated in MATLAB using the PsychToolbox (Brainard, 1997; Pelli, 1997). They consisted of drifting bar apertures at various orientations, which exposed a checkerboard pattern at $100 \%$ contrast moving parallel to the bar orientation (Dumoulin and Wandell, 2008). Alternating rows of checks moved in opposite directions. The checkerboard motion direction reversed at random intervals, with a minimum of $4 \mathrm{~s}$ between reversals. The bar width subtended one-quarter of the stimulus radius $\left(1.56^{\circ}\right)$, and this was also the spatial frequency of the checkerboard. The bar moved across the stimulus aperture in 20 evenly spaced steps, each $0.625^{\circ}, 1 / 20$ th of the stimulus window diameter. As there was one step at the start of each functional volume acquisition, each pass of the stimulus took 20 TRs, 30 s. Four bar orientations and two different motion directions for each bar were presented, giving a total of eight bar motion directions (upward, downward, left, right, and four diagonals) within each scan. After each horizontal or vertical bar orientation pass, $30 \mathrm{~s}$ of mean-luminance (zero contrast) stimulus were displayed. As diagonal bar orientations were alternated with horizontal/vertical orientations, four mean-luminance blocks were presented at regular intervals during the scan.

Subjects fixated a dot in the center of the visual stimulus. This changed colors between red and green at random intervals. To ensure attention was maintained subjects pressed a button on a response box every time the color changed, which was on average every $3 \mathrm{~s}$, with a minimum change interval of $1.8 \mathrm{~s}$. Scans where performance on this task dropped below $75 \%$ were discarded (two scans of one subject).
Eye movement recording. Eye movements during stimulus presentation will affect the pRF sizes estimated, as with any RF measurement, because without accurate fixation the part of the retina activated by the stimulus will not be as expected. Most subjects were experienced in visual fMRI or psychophysics experiments requiring accurate fixation, and so performance on the task presented at fixation typically exceeded $90 \%$. Subjects were also instructed on the importance of maintaining fixation.

Eye movements for four of the subjects used in the study were measured outside of the scanner using a highly accurate Eyelink II system (SG Research). This allowed the distribution of fixation positions to be measured with the same stimulus and task as was shown in the scanner. Values obtained during moving bar presentations and the meanluminance blank periods were not significantly different, and so presentation of the moving bar did not appear to affect eye movements.

Preprocessing of anatomical and functional images. fMRI analysis was performed in the mrVista software package for MATLAB, which is freely available at (http://white.stanford.edu/software/). T1-wieghted anatomical scans were resampled to $1 \mathrm{~mm}^{3}$ resolution. The resulting anatomical image was automatically segmented using FSL (Smith et al., 2004) and then hand-edited to minimize segmentation errors (Teo et al., 1997). The cortical surface was reconstructed at the gray-white matter border and rendered as a smoothed 3D surface (Wandell et al., 2000). Head movement and motion artifacts between and within functional scans were measured and corrected for (Nestares and Heeger, 2000). Functional data were then averaged across scans. Functional data were aligned to anatomical scans (Nestares and Heeger, 2000) and interpolated to the anatomical segmentation.

$f M R I$ data analysis. Population receptive field sizes and positions were estimated from the fMRI data and visual stimulus position time course (Dumoulin and Wandell, 2008). The BOLD response of each voxel was predicted using a two-dimensional Gaussian pRF model. This modeled the center location ( $x$ and $y$ parameters) and spread $(\sigma)$ of the most responsive position of the voxels to the stimulus. The predicted fMRI time course was calculated by convolution of the modeled pRF, the stimulus sequence, and a canonical BOLD hemodynamic response function (HRF) (Eq. 1) (Friston et al., 1998; Glover, 1999; Worsley et al., 2002). The pRF parameters for each voxel are determined by minimizing the sum of squared errors (RSS) between the predicted and observed fMRI time series.

After estimating the pRF parameters, the HRF parameters were determined by minimizing the RSS between the predicted and observed BOLD responses over the entire recorded cortex where the pRF model explained $>10 \%$ of the variance in the data. During this procedure, $\mathrm{pRF}$ parameters were kept constant. The starting point was a canonical twogamma HRF (Friston et al., 1998; Glover, 1999; Worsley et al., 2002) as follows:

$h(t)=\left(t / d_{1}\right)^{a_{1}} \exp \left(-\left(t-d_{1}\right) / b_{1}\right)$

$$
-c\left(t / d_{2}\right)^{a_{2}} \exp \left(-\left(t-d_{2}\right) / b_{2}\right)
$$

where $h$ is the hemodynamic response, $t$ is time in seconds, $d_{j}=a_{j} b_{j}$ is the time to the peak, $a_{1}=6, a_{2}=12, b_{1}=b_{2}=0.9 \mathrm{~s}$, and $c=0.35$. These parameters were iteratively searched to minimize the RSS. The parameter combinations were constrained to preserve the overall HRF profile [i.e., one initial positive peak $\left(d_{1}<d_{2}\right)$ followed $(c \geq 0)$ by a late negative undershoot]. Next, the pRF parameters were refined to optimize the pRF parameters given this HRF. The pRFs were sampled by bars traveling in opposite directions, a technique also used during conventional retinotopic mapping to account for the hemodynamic delay (Smith et al., 2001). Therefore, the HRF fitting procedure should find the optimal parameters by fitting responses to both directions.

PRF models derived using the canonical two-gamma HRF, without HRF fitting, yield slightly different absolute values but our main findings remain. Similarly, using a difference of Gaussian pRF model to incorporate the effects of a suppressive surround (Zuiderbaan et al., 2010), again yields slightly different values but the same pattern of results. Therefore, our results are robust to changes in modeling methods.

The polar angle and eccentricity maps of the pRF centers of each voxel were rendered onto an inflated cortical surface (Wandell et al., 2000), and 
the positions of $\mathrm{V} 1$ and other visual areas were determined and defined as regions of interest (ROIs) by relation to visual field representation (Sereno et al., 1995; Wandell et al., 2007). Areas of low mean fMRI signal, corresponding to pial draining veins (Olman et al., 2007; Winawer et al., 2010), were excluded from subsequent analysis (except for the computation of the V1 surface area).

We determined the CMF for every voxel on the gray-white matter border independently. All analysis of pRF, CMF, and point image was therefore restricted to voxels on the gray-white matter border. Gray matter thickness was ignored, effectively treating the gray-white matter border as the cortical surface. At each cortical location, we computed the distance (in millimeters) to neighboring locations along the cortical surface. To compute the CMF, this cortical distance was divided by the change in preferred pRF location (degrees of visual angle) of the same cortical locations. Neighboring voxels with poor pRF model fits (variance explained $<30 \%$ ) were removed from this computation, as were voxels outside the ROI. The population point image was computed by multiplying the CMF and pRF size estimates for each cortical location. This technique estimates the CMF and point image for each individual cortical location.

$p R F$ sensitivity and bias. For these particular stimulus and scanning parameters, we produced a forward model to determine the sensitivity range. The forward model estimated the fMRI time series elicited by a given $\mathrm{pRF}$. Assuming independent white noise, the sensitivity to changes in pRF size is proportional to the changes in the variance of the predicted fMRI time series. The sensitivity range was determined by the difference in the predicted fMRI time series elicited by small changes in pRF size parameters (1-10\% of the pRF size). The forward model predicted the largest fMRI signal changes, within $5 \%$ of the maximum, when pRF sizes were perturbed between 0.45 and $2.6^{\circ}$. Thus, given this particular stimulus, the sensitivity was within $5 \%$ of the maximum for pRF sizes between 0.45 and $2.6^{\circ}$, and within $50 \%$ of the maximum for pRF sizes between 0.16 and $5.2^{\circ}$. This sensitivity range is optimal for the pRF sizes previously reported in early human visual cortex (Dumoulin et al., 2003; Amano et al., 2009; Winawer et al., 2010), and those measured here.

To assess a possible bias in the pRF size analysis, we simulated fMRI time series with known pRF sizes and positions. We distributed 90,000 pRFs equally throughout the visual field with pRF sizes between 0.05 and $6^{\circ}$. The simulated time series were created by convolving each pRF with the stimulus aperture and a canonical HRF (Eq. 1). We added Gaussian white noise to the simulated time series and then ran the pRF data analysis to compare the estimated pRF to the actual pRF. Signal-to-noise ratio (SNR) levels were manipulated to a conservative level (50\% average variance explained) and that of the actual fMRI data presented in this paper ( $73 \%$ average variance explained). There is indeed a bias when the pRF sizes approach the edges of the sensitivity ranges (i.e., very small and very large pRFs). This bias is influenced by the SNR. We believe the bias is mediated by a skewed pRF size distributions around the true pRF size value when the SNR decreases. This skew is introduced because pRF sizes cannot go below zero (unavoidable bias at very small pRF sizes) nor go beyond an upper pRF size limit (fixed analysis boundary). The simulations show, however, that at the typical SNR and pRF sizes of our data these biases are negligible.

Fits of pRF size, CMF, and population point image versus eccentricity. The $\mathrm{pRF}$ size versus eccentricity relationship was described by the following equation:

$$
y=a x+b,
$$

where $y$ is the pRF sizes, $x$ is eccentricity, and $a$ and $b$ are the slope and intercept respectively. The $a$ and $b$ terms were estimated by minimizing the RSS to the eccentricity-binned data, with each error weighted by the inverse of the SEM in that bin. In a similar fashion, the CMF versus eccentricity function was described by the following equation, also used in other studies (Engel et al., 1994; Sereno et al., 1995; Dougherty et al., 2003; Schira et al., 2010):

$$
y=\frac{1}{c x+d}
$$

where $y$ is CMF and $x$ is eccentricity. As population point image is the product of $\mathrm{pRF}$ and $\mathrm{CMF}$, its relationship with eccentricity could be described as the product of Equations 2 and 3, as follows:

$$
y=\frac{a x+b}{c x+d}
$$

where $y$ is population point image and $x$ eccentricity. For a constant population point image across all eccentricities, this formulation proscribes a set of properties of the pRF size and CMF functions described in Equations 2 and 3: $a / b$ must be equal to $c / d$. This situation describes a fixed ratio between the slope and intercept terms in pRF size versus eccentricity and $1 / \mathrm{CMF}$ versus eccentricity.

However, we used Equation 3 to describe the point image changes as a function of eccentricity. The fit of Equation 3 is only a description of the data. Using Equation 2 or 4 yielded similar results (i.e., decrease of point image with eccentricity). We favor Equation 3 because, first, it gives a significantly better fit than Equation 2 in hV4 and LO1 (it captured the curve). Second, there are no significant improvements in fit by using Equation 4. Furthermore, Equation 3 avoids the excess of free parameters associated with Equation 4 and provides a single solution, whereas Equation 4 can potentially describe the same relationship with different sets of parameters. Finally, Equation 4 does not allow the distribution of the slope terms to be examined (as in Fig. 1D) in any straightforward way, and any change in one slope term can be counteracted by changes in other terms.

\section{Results}

\section{Individual hemisphere data shows considerable variation in} pRF size and CMF, but similar point images

We determined the individual CMF, pRF size, and population point image for area $\mathrm{V} 1$, for all hemispheres of 11 subjects, with two hemispheres shown as examples (Fig. $1 A-C$ ). The pRF size increased with visual field eccentricity (Fig. 1A; 20 of 22 hemispheres), and the CMF decreased with eccentricity (Fig. $1 B ; 21$ of 22 hemispheres). Those hemispheres in which pRF size and CMF changes did not follow expected trends were from the same subject. The population point image had a slope (the $c$ term in Eq. 3) that was not significantly different from zero in 9 of 22 hemispheres between 1.0 and $5.5^{\circ}$ (Fig. $1 C, D$ ), determined by bootstrapping the binned data and refitting. In 11 hemispheres, this term was significantly greater than zero (point image decreased with increasing eccentricity), and in 2 hemispheres, it was significantly less than zero (point image increased with eccentricity). The mean $c$ term in Equation 3 for the fit to point image bins from individual subjects was 0.018 .

There are many technical issues that may prevent accurate reconstruction of the $\mathrm{pRF}$ size and CMF slope, and these issues are multiplied in the computation of the population point image. In many hemispheres in which population point image was very different from constant, changes in population point image are associated with irregularities in the modeled visual field representation, leading to unusual variation of CMF or pRF size. Therefore, we suspect a methodological rather than biological reason for finding a far from constant population point image in certain hemispheres. However, overall, the data from individual hemispheres support the conclusion of a slight decrease in V1 point image with eccentricity.

\section{Population point image depends on $\mathrm{pRF}$ size and CMF being measured in the same hemisphere}

CMF and pRF sizes vary by a factor of at least 2 between subjects (Dougherty et al., 2003; Dumoulin and Wandell, 2008) (Fig. $1 A, B)$. Despite this individual variation in $\mathrm{pRF}$ size and CMF, population point image remains relatively constant between 

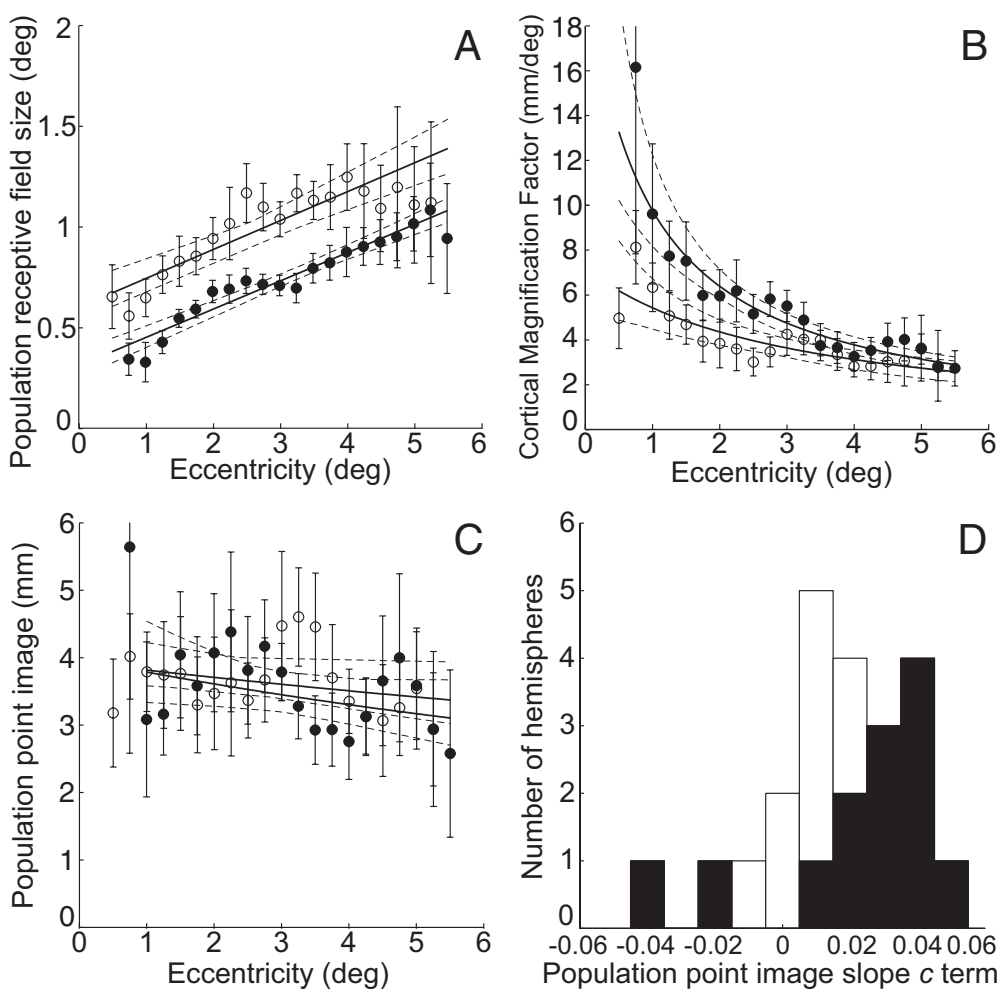

Figure 1. Changes of pRF size, CMF, and population point image size across visual field eccentricity in V1 voxels from the left hemispheres of two subjects (one shown in black, the other in white). $\boldsymbol{A}$, pRF size increases with visual field eccentricity. $\boldsymbol{B}$, CMF decreases with visual field eccentricity. C, Population point image size is near constant, decreasing slightly with eccentricity. Although pRF size and CMF are significantly different between these hemispheres, the population point image is very similar. Error bars reflect the SEM of the binned points, corrected for upsampling in the analysis. The solid lines reflect the best-fitting functions as described in Equations $2(\boldsymbol{A})$ and $3(\boldsymbol{B}, \boldsymbol{C})$ for fits to those binned points, and the dashed lines reflect $95 \%$ confidence intervals of these fits and were determined by bootstrapping the binned data and refitting. $\boldsymbol{D}$, Histogram of population point image slopes terms for all 22 hemispheres. The black points represent slopes from 13 hemispheres that were significantly different from zero by bootstrapping.
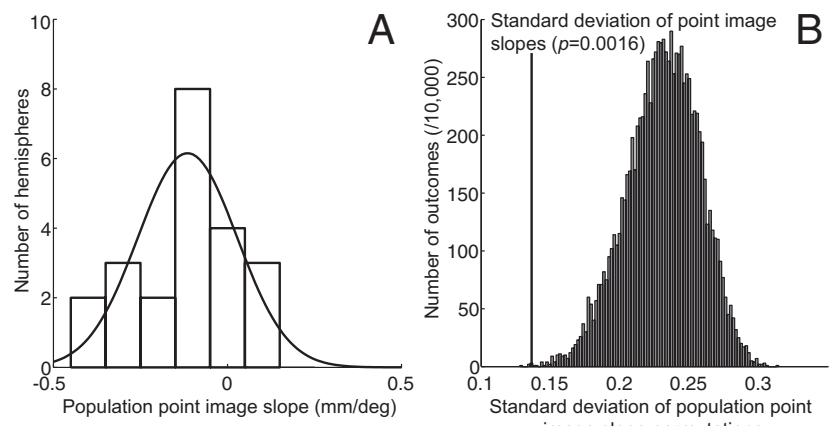

Figure 2. $\quad \boldsymbol{A}$, Histogram of population point image slopes at $4^{\circ}$ visual field eccentricity for all 22 hemispheres, determined as the product of pRF and CMF fits. The best-fitting Gaussian function to this distribution, used in $\boldsymbol{B}$, is also shown. $\boldsymbol{B}$, SD distribution of 10,000 random permutations of pRF and CMF slope products (gray histogram), compared with the SD seen when pRF and CMF slopes were always taken from the same hemispheres (black line). When hemisphere relationships are maintained, the SD is significantly smaller than the distribution determined by random permutations ( $p=0.0016$, two-tailed).

hemispheres as a high pRF size is typically found with a low CMF and vice versa (Fig. 1). To examine whether the $\mathrm{pRF}$ size and CMF slopes covary within hemispheres to give rise to a near-constant population point image, we randomly permutated the $\mathrm{pRF}$ and CMF slopes between hemispheres. If $\mathrm{pRF}$ and CMF in individual hemispheres covaried, the distribution of randomly permuted population point image slopes should be significantly broader than that found when the relationship between measures from each hemisphere was maintained.

In the preceding data analysis, $\mathrm{pRF}$, $\mathrm{CMF}$, and population point image were computed for each individual cortical location. That approach cannot be used to permute $\mathrm{pRF}$ and CMF values between hemispheres. Therefore, to randomly permute the $\mathrm{pRF}$ and CMF values, we computed the population point image slope from the fitted lines to the $\mathrm{pRF}$ and CMF values at $4^{\circ}$ eccentricity (Fig. 2). The individual subject fits for $\mathrm{pRF}$ and CMF (Fig. $1 A, B$ ) were multiplied, resulting in a function following Equation 4. As this function has many parameters, its slope at $4^{\circ}$ was measured for each hemisphere (Fig. $2 A$ ). This distribution of the population point image slopes was computed 10,000 times, with random pRF and CMF combinations. Figure $2 B$ shows the SD distribution of these 10,000 random permutations of slope products, with the line representing the $\mathrm{SD}$ when both measures are always taken from the same hemisphere. Compared with random permutations, the probability of the hemisphere-specific combination of $\mathrm{pRF}$ and $\mathrm{CMF}$ is $p=0.0016$ (two-tailed, measured as the number of permuted SDs that is smaller than when the measures are taken from the same hemisphere). This probability demonstrates that the distribution of population point image slopes between hemispheres depends on both properties being measured in the same hemisphere. In other words, the pRF and CMF combinations are unique for each hemisphere.

The same analysis performed for later visual areas showed the probability of the hemisphere-specific combination was still significant for V2 $(p=0.039)$, but not significant for V3 $(p=0.076)$, hV4 $(p=0.249)$, or LO1 $(p=0.119)$.

\section{V1 size, $\mathrm{pRF}$ size, $\mathrm{CMF}$ covary between hemispheres}

PRF size, CMF, and V1 sizes also vary by a factor of at least 2 between subjects (Stensaas et al., 1974; Andrews et al., 1997; Dougherty et al., 2003; Duncan and Boynton, 2003; Dumoulin and Wandell, 2008). It may be that these covary, such that a change in one property is associated with a change in the others.

Here, we examined the correlations between pRF size and CMF (both measured at $4^{\circ}$ of visual field eccentricity), and V1 size to see whether there is a relationship between them across hemispheres. The V1 size was measured along the gray-white matter boundary between 2.5 and $5.5^{\circ}$ of visual field eccentricity. As V1 size is an area, and CMF and pRF size are linear measures, CMF was squared to give CMF area. pRF size $(\sigma)$, effectively a radius, was squared and multiplied by $\pi$ to calculate the pRF area.

All three measures are strongly correlated between hemispheres (Fig. 3). There is a strong correlation between $\mathrm{V} 1$ area and CMF area across hemispheres $(r=0.788 ; p=0.00001)$ (i.e., when the CMF is larger, more area of visual cortex is required to rep- 
resent the same area of visual field) (Fig. $3 A$ ). As V1 area and CMF area both measure the area of cortex that represents a given part of the visual field, this correlation is expected. It gives an idea of the maximum correlation possible in these data, given the level of noise in the data.

There is strong negative correlation between pRF area and CMF area across hemispheres $(r=-0.603 ; p=0.003)$, so when a hemisphere has smaller pRF sizes, it also has a larger cortical magnification factor (Fig. 3B). We found no significant correlation between pRF area and CMF area for V2 $(r=-0.248 ; p=0.266)$, V3 $(r=-0.381 ; p=0.079), \operatorname{hV} 4(r=$ $-0.282 ; p=0.204)$, or LO1 $(r=-0.251$;

$p=0.258$ ), although trends were present. Last, we report a strong negative correlation between V1 surface area and pRF area across all hemispheres $(r=-0.613 ; p=0.002)$ (i.e., as the V1 surface area increases the $\mathrm{pRF}$ area decreases) (Fig. $3 C$ ). Thus, with bigger visual field representation on the cortical surface, measured by either V1 surface area or CMF, pRF sizes decrease.

\section{V1 population point image varies less between hemispheres than CMF and pRF size}

A constant population point image between hemispheres and subjects may underlie the correlations between V1 size, CMF, and pRF size. If this is the case, the variation in population point image sizes should be smaller than the variation in these other properties.

We examined whether the population point image varies less than the pRF size, CMF, and V1 size. Again, because V1 size is an area, the CMF, pRF, and population point image sizes were converted to area terms. Comparisons were also made for linear measurements, excluding V1 area.

Table 1 shows the mean value of each measure and the corresponding SD. The values were normalized by subtracting and then dividing by the mean, and the SD of these normalized values was determined. The normalized population point image variance is significantly less than those of the CMF and V1 size measures, although not significantly less than $\mathrm{pRF}$ size. These results are the same for both for both linear and area data. The probability of each outcome by chance is given in the final column, measured using a one-tailed Pitman's test for paired comparisons of variance (Pitman, 1939).

We also compared the variances of linear pRF size, CMF, and population point image in later visual areas. The variance of the population point image was often significantly greater than that of pRF size and CMF. As population point image is the product of pRF size and CMF, both of which vary between subjects, a greater variance in population point image would be expected if $\mathrm{pRF}$ size was not related to CMF. Population point image varied significantly more than pRF size in V3 $(p=0.036), \operatorname{hV} 4(p=0.038)$, and LO1 $(p=0.012)$, and significantly more than CMF in V2 $(p=0.015)$.

\section{Data grouped across subjects show population point image decreases slightly with eccentricity in V1, and much more in later areas}

Useful analysis of the changes in pRF size, CMF, and point image across the visual processing hierarchy requires a clearer signal than was found in our individual subject data. We therefore grouped together all voxels in areas $\mathrm{V} 1, \mathrm{~V} 2, \mathrm{~V} 3, \mathrm{hV} 4$, and LO1 to give a stronger data set.

Comparable with previous reports, pRF size increased with visual field eccentricity and up the visual processing hierarchy (Fig. 4A) (Dumoulin and Wandell, 2008; Kay et al., 2008; Amano et al., 2009; Winawer et al., 2010). CMF decreased with eccentricity in all visual areas (Fig. $4 B$ ) (Sereno et al., 1995; Dougherty et al., 2003; Dumoulin and Wandell, 2008; Kay et al., 2008). The CMF of V1 increased less in the central visual field representation than those of other areas, as previously reported (Schira et al., 2009). The CMFs of V2 and V3 were very similar, as were the CMFs of hV4 and LO1 (Fig. $4 B$ ). At eccentricities greater than $1.5^{\circ}, \mathrm{CMFs}$ of $\mathrm{hV} 4$ and LO1 were significantly smaller than those of V2 and V3, reflecting the smaller size of hV4 and LO1, and a relatively greater representation of the central visual field in the areas (center bias), as previously reported (Ejima et al., 2003; Brewer et al., 2005; Larsson and Heeger, 2006).

The population point image of V1 decreases slightly with eccentricity from 1.0 to $5.5^{\circ}$ by $0.2 \mathrm{~mm}$ per degree (Fig. $4 C$ ). Similarly to mean of the individual subject data (Fig. $1 D$ ), the $c$ term in Equation 3 was 0.019 for $V 1$, and was significantly different from zero, determined by bootstrapping the binned data and refitting.

The population point image of $\mathrm{V} 2, \mathrm{~V} 3, \mathrm{hV} 4$, and LO1 decreases considerably with eccentricity. This change from V1 to V2 has been reported in nonhuman primates (Rosa et al., 1988). This decrease is greater for hV4 and LO1 than for V2 and V3. The differences between these point image slopes can be attributed to differences between both CMFs and pRFs.

Equation 4 also allows us to examine the conditions of the change in pRF size with eccentricity under which population point image might have been constant given the measured CMF of V1. As discussed in Materials and Methods, a constant population point image requires the ratios of slopes and intercepts for pRF size versus eccentricity and 1/CMF versus eccentricity to be the same $(a / b=c / d)$. Figure $4 A$ gives $a / b$ as 0.31 (95\% confidence intervals, $0.27-0.34)$, while Figure $4 B$ gives $c / d$ as 0.64 (0.540.77 ). Therefore, this test confirms a nonconstant relationship between $\mathrm{pRF}$ size and CMF.

The effect of eye movements can be factored out of pRF size measurements by fitting a Gaussian distribution to eye positions. The average SD of eye positions is $0.22^{\circ}$ for the four subjects measured. But, because there is inevitably some noise in the measurement of eye positions, this value represents an upper bound of the actual variation of fixation positions. Deconvolving this Gaussian with the pRF size gives an estimate of the pRF size 
Table 1. Analysis of the variation across hemispheres in pRF size, CMF, and population point image (both linear and as areas) and V1 surface area, each measured at $4^{\circ}$ visual field eccentricity

\begin{tabular}{|c|c|c|c|c|}
\hline & Property (unit) & Mean $( \pm S D)$ & $\begin{array}{l}\text { Normalized SD } \\
{[\mathrm{SD}(\text { values }- \text { mean)/mean) }]}\end{array}$ & $\begin{array}{l}\text { Significance level (H0: variance } \leq \text { variance } \\
\text { of population point image) }\end{array}$ \\
\hline \multirow[t]{4}{*}{ Area measures } & pRF area $\left(\mathrm{deg}^{2}\right)$ & $3.71( \pm 0.82)$ & 0.2209 & 0.131 \\
\hline & CMF area $\left(\mathrm{mm}^{2} / \mathrm{deg}^{2}\right)$ & $9.92( \pm 2.49)$ & 0.2514 & 0.025 \\
\hline & V1 area $\left(\mathrm{mm}^{2}\right)$ & $218.33( \pm 61.47)$ & 0.2815 & 0.011 \\
\hline & Population point image area $\left(\mathrm{mm}^{2}\right)$ & $32.53( \pm 5.59)$ & 0.1718 & \\
\hline \multirow[t]{3}{*}{ Linear measures } & pRF size (deg) & $1.08( \pm 0.12)$ & 0.113 & 0.103 \\
\hline & CMF (mm/deg) & $3.13( \pm 0.39)$ & 0.1241 & 0.024 \\
\hline & Population point image size (mm) & $3.21( \pm 0.27)$ & 0.085 & \\
\hline
\end{tabular}

The normalized variance of population point image is significantly less than that of CMF and V1 size, compared using one-tailed Pitman's test for paired comparison of variance. However, comparisons to pRF size do not reach significance. Population point image is therefore relatively constant between different hemispheres from different subjects. This table also provides a mean measure for the size and area of the population point image.

A

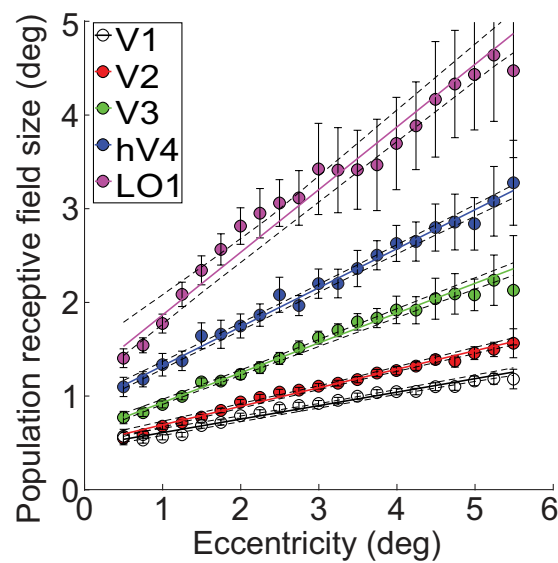

B

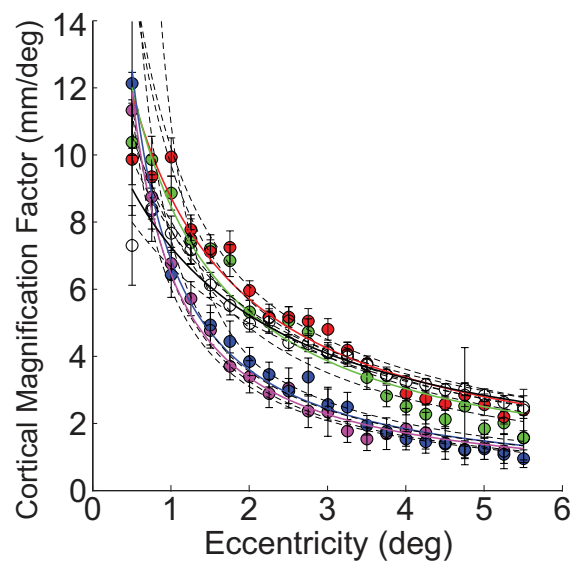

C

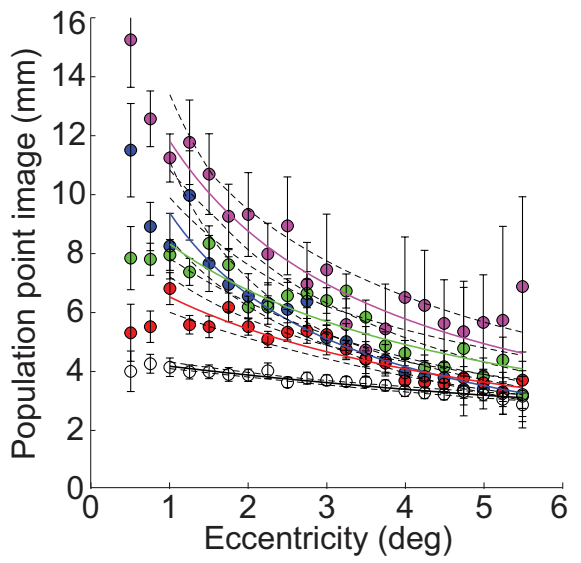

Figure 4. Changes of pRF size and CMF and population point image size across visual field eccentricity in voxels of areas V1, V2, V3, hV4, and L01, grouped from all 22 hemispheres. $\boldsymbol{A}$, pRF size increases with visual field eccentricity and up the visual processing hierarchy. $\boldsymbol{B}, \mathbf{C M F}$ decreases with visual field eccentricity. $\boldsymbol{C}$, Population point image size is near constant, decreasing slightly with eccentricity in V1. In later visual areas, it is far from constant, decreasing considerably with eccentricity. Error bars reflect the SEM of the binned points, corrected for upsampling in the analysis. The solid lines reflect the best-fitting functions as described in Equations $2(\boldsymbol{A})$ and $3(\boldsymbol{B}, \boldsymbol{C})$ for fits to those binned points, and the dashed lines reflect $95 \%$ confidence intervals of these fits and were determined by bootstrapping the binned data and refitting.

without eye movements (Levin et al., 2010). This results in a pRF size $a / b$ ratio of 0.35 ( $95 \%$ confidence interval, $0.30-0.39$ ), which is still outside the range that might indicate constancy $(c / d, 0.54-$ 0.77 ). Therefore, eye movements have not caused the population point image in V1 to deviate from constancy.

\section{Receptive field sizes in V2 and V3 can be explained by a constant sampling from V1}

Considering that later visual areas derive their visual field representations from those in early visual areas, it is not surprising that their point image is not constant when expressed in visual space. But when point image is expressed relative to preceding cortical processing stages, it may be constant. When the $\mathrm{pRF}$ is expressed in terms of a cortical visual field representation, we refer to it as corticocortical pRF (cc-pRF).

In a simple theoretical treatment of this corticocortical sampling, a later area $(\mathrm{Vn})$ will inherit much of its $\mathrm{pRF}$ size directly from V1. As most visual areas are known take inputs from more than one previous area, and receive feedback connections, this is an oversimplification. Nevertheless, if we assume that both V1 and Vn pRF sizes and the corticocortical sampling may be described by Gaussians and that Vn samples only from V1, the Vn pRF size can be computed by a convolution of $\mathrm{V} 1 \mathrm{pRF}$ size with the corticocortical sampling size (cc-pRF) as follows:

$$
\sigma_{\mathrm{Vn}}^{2}=\sigma_{\mathrm{V} 1}^{2}+\sigma_{\mathrm{Vn} \mid \mathrm{V} 1}^{2}
$$

where $\sigma_{\mathrm{Vn}}$ is the pRF size, $\sigma_{\mathrm{V} 1}$ the pRF size of $\mathrm{V} 1, \sigma_{\mathrm{Vn} \mid \mathrm{V} 1}$ reflects the size of the sampling in visual space (i.e., the cc-pRF from V1 to Vn). Thus, we can compute the cc-pRF by the following:

$$
\sigma_{\mathrm{Vn} \mid \mathrm{V} 1}^{2}=\sqrt{\sigma_{\mathrm{Vn}}^{2}-\sigma_{\mathrm{V} 1}^{2}}
$$

These cc-pRFs relative to V1 are shown in Figure 5A. The cc-pRF sizes in degrees of visual angle follow a very similar pattern to $\mathrm{pRF}$ sizes.

We can convert the cc-pRF size estimates from visual space units (degrees) to cortical space units (millimeters) by multiplying them by the CMF of V1. These estimates are shown in Figure $5 B$. The cc-pRFs in millimeters of V1 space do not vary significantly as a function of eccentricity in V2 and V3 (i.e., the slopes did not differ significantly from zero). Likewise, the $a / b$ ratio fit using Equation 2 for the data in Figure $5 A$ was 0.63 and 0.55 for V2 and V3, respectively. Both ratios fall within the $95 \%$ confidence intervals of the CMF $c / d$ ratio $(0.54-0.77)$, indicating a constant relationship. In $\mathrm{hV} 4$, there is a slight decrease with eccentricity, whereas in LO1 there is a considerable decrease with eccentricity. For hV4 and LO1, the $a / b$ ratios were 0.47 and 0.41 , respectively, both outside the range that indicates constancy.

When we used the same method to determine how each area might sample from other visual areas (we only considered areas with smaller pRFs), no other possible combination resulted in a constant sampling extent across eccentricity. This suggests a con- 
A
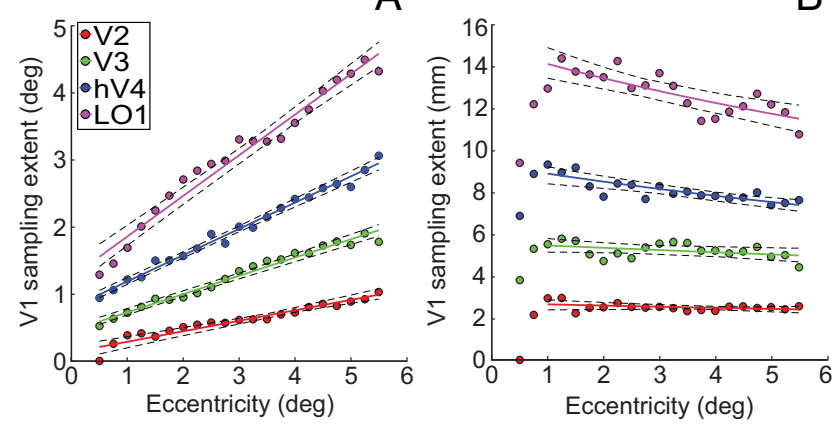

Figure 5. Population receptive field size differences between visual areas and measures of the cc-PRF size of $\mathrm{Vn}$ from V1, grouped from all 22 hemispheres using the binned data in Figure 4. A, PRF size differences (following Eq. 6) compared with V1 increased with eccentricity, increased up the visual processing hierarchy, and increased in slope up the hierarchy. $\boldsymbol{B}$, Multiplying pRF size differences by the CMF of $\mathrm{V} 1$ yields the corticocortical pRF size of Vn from V1. This does not vary with eccentricity for V2, or V3, but decreases slightly with increasing eccentricity in $\mathrm{hV} 4$ and considerably in L01. The size increases up the visual processing hierarchy. Lines were fit to bins with Equation $2(\boldsymbol{A})$ or Equation $3(\boldsymbol{B})$. Bins were bootstrapped and fits repeated to give $95 \%$ confidence intervals (dashed lines), and significant differences from constancy were examined using the slopes of these confidence intervals.

stant cortical sampling size of V1 across eccentricity by both V2 and $\mathrm{V} 3$, and that this is unique to sampling from $\mathrm{V} 1$.

\section{Discussion}

\section{Summary}

Our results show that population point image in V1 is near constant, decreasing slightly with eccentricity. This decrease has a narrow distribution between hemispheres. This depends on both pRF size and CMF being measured in the same hemisphere, as both vary considerably between hemispheres. Variations in pRF size, CMF, and V1 surface area are correlated. Furthermore, the variance of population point image measurements is significantly less than that CMF and V1 area, although not significantly less than that of pRF size. This suggests a relatively constant point image between hemispheres and subjects, as well as across the V1 surface.

In later visual areas, population point image decreases considerably with eccentricity. This decrease becomes more pronounced up the visual processing hierarchy. However, when the pRFs here are expressed in V1 cortical surface area (i.e., cc-pRF), they are constant in V2 and V3, and near constant in hV4. Thus, these later visual areas pool from a constant surface area of V1, regardless of eccentricity.

\section{Relationship to previous studies}

The concept of point image, the cortical representation of one RF size, is adapted from neurophysiological studies to give population point image, the cortical representation of one pRF size. Point image is equivalent to the area of cortex activated by a point in visual space, although population point image is not, as it is based on an aggregate RF. Neurophysiological studies differ, proposing a constant relationship between CMF and RF size (Hubel and Wiesel, 1974; Albus, 1975), decreasing point image with increasing eccentricity in the foveal representation (Dow et al., 1981), or decreasing point image with eccentricity to a minimum at $5.5^{\circ}$, followed by a slight increase with eccentricity (Van Essen et al., 1984). We find large variability between hemispheres, and speculate that limited sample sizes and combinations of different hemispheres may explain these variable results. However, our results are most reliable between 1.0 and $5.5^{\circ}$ eccentricity, so we may miss considerable variation within the foveal representation or across larger eccentricity ranges. Meta-analysis of neurophysiological studies found a slight decrease with eccentricity (McIlwain, 1986). This result is supported by our data.

Our data extend observations to V2, V3, hV4, and LO1, and show an increasing change of population point image with eccentricity up the processing hierarchy. Increasing point image slope from V1 to V2 is consistent with neurophysiological results (Rosa et al., 1988).

\section{Constancies in cortical architecture}

Constant point image would suggest constancies in the neural architecture processing visual information. The concept of point image is related to the concept of a hypercolumn, a repeating unit containing neurons with a complete range of response selectivities (Hubel and Wiesel, 1974). But the absolute sizes differ and a point image probably includes several hypercolumns (Hubel and Wiesel, 1977; Dow et al., 1981; Van Essen et al., 1984). Point image constancy might reflect constant factors in intra-area cortical architecture, such as the horizontal distance of cortical cell interconnections, or interarea cortical architecture, for example that a constant density of optic radiation fibers reaching V1 (le Gros Clark, 1941) determines both CMF and pRF sizes.

If RF sizes were closely linked to intra-area architecture, this should also be true in later areas, predicting a constant point image in other visual areas. Our results do not support this view. Instead, we find that pRF sizes in later areas are related to V1 CMF. This is also seen in neurophysiological results, in which V4 neurons all have similarly sized circular RFs when expressed on the V1 surface (Motter, 2009), despite large changes in RF shape and size in visual space. Therefore, rather than close linking between $\mathrm{pRF}$ size and intra-area architecture, we propose that $\mathrm{pRF}$ sizes in V2, V3, and to some degree hV4, are determined by constant sampling of the V1 visual field map (i.e., by interarea architecture).

Similarly, constant sampling of visual space (point image) by V1 might not be expected considering the processing steps between visual space and V1 (Perry and Cowey, 1985). The retinal area devoted to each degree of visual space decreases with visual field eccentricity because the center of curvature of the retina is behind the posterior nodal point of the lens. Furthermore, macaque retinal ganglion cell density decreases slightly with eccentricity. Finally, the lateral geniculate nucleus (LGN) devotes more of its neuronal population to the central visual field than the retina does (Perry and Cowey, 1985).

Consequently, the slight decrease in V1 may result from constant sampling of the center-biased visual field representation of the LGN by V1. Any difference between RF sizes in LGN and V1 may be constant when the magnification factor of LGN is taken into account. In support of this argument, Andrews et al. (1997) find that the sizes of V1 and LGN are correlated between subjects, which implies a correlation between V1 pRF size and LGN magnification factor. In addition to these proposed constancies in cortical architecture within subjects, our results also suggest that this cortical architecture is similar between subjects despite variations in overall size of $\mathrm{V} 1$.

\section{Technical considerations}

Unlike in neurophysiology, measuring point image from $\mathrm{AMRI}$ is straightforward because many recording points are acquired in parallel. Determining point image changes across V1 neurophysiologically requires hundreds of recording points in 
each hemisphere. Examining individual differences requires thorough characterization of many animals. In contrast, pRF mapping requires less than one hour of scanning per subject. Once pRF models are computed (Dumoulin and Wandell, 2008), population point image, CMF, and V1 surface area can be calculated quickly. This fMRI-based approach is therefore attractive when examining changing response properties across the cortical surface and between individuals.

Single-neuron measurements of preferred RF visual field position are subject to the scatter of positions represented at any cortical location (McIlwain, 1986), so single-neuron measurements add noise to CMF and therefore point image computations. Multiunit recordings of the aggregate RF of this population avoid this issue. This concept is similar to our population RF measure.

The pRF combines neuronal RFs, extraclassical RF effects, nuisance factors, and, importantly, the spread of neuronal RFs within the fMRI recording site (for review, see Smith et al., 2001; Dumoulin and Wandell, 2008). If these factors are not approximately proportional to RF size, measuring relationships between pRF size and CMF will give different results than measuring relationships to RF size and CMF. However, neurophysiological data suggest that RF scatter is proportional to RF size (Hubel and Wiesel, 1974; Dow et al., 1981; Gattass and Gross, 1981; Albright and Desimone, 1987; Fiorani et al., 1989; Hetherington and Swindale, 1999). However, an fMRI recording site has a greater spatial extent on the cortical surface. The visual field extent represented in an fMRI recording site will change with CMF. Therefore, we must ask whether the relationships we find between pRF and CMF result from inherent dependence. We do not believe this explanation fits well with our results. First, we do find a small decrease in point image with eccentricity in V1, similar to a metaanalysis of neurophysiological results (Mcllwain, 1986). Second, we find large changes in population point image across eccentricity in later areas, with systematic changes up the visual processing hierarchy. Third, if between-hemisphere correlations resulted from inherent dependence of pRF sizes on the CMF, this correlation should be as clear in later areas, which is not the case. Fourth, we find a constant relationship between CMF and pRF size changes when expressed in V1 area (cc-pRFs). But this relationship relies on measurements from the same visual field position across visual field maps (i.e., in different recording sites). Therefore, it seems unlikely that these findings should have a methodological basis.

\section{Perception}

These results extend links between V1 CMF and visual acuity (Duncan and Boynton, 2003) and between V1 size and perceived object size (Schwarzkopf et al., 2011). The corresponding pRF size changes may underlie these results. Duncan and Boynton (2003) showed that larger CMFs correlate with higher visual acuity. As we show that larger CMFs correspond to smaller pRF sizes, this higher acuity may result from smaller RF sizes and so more detailed analysis of the visual field. Schwarzkopf et al. (2011) found that larger V1 sizes correlate with weaker illusory effects on perceived object size. We show larger V1 sizes correspond to smaller pRF sizes and larger CMF-although similar point image. Therefore, the weaker illusory effects may result from smaller RF sizes separated by greater cortical distances for the same visual field distances. This should reduce the visual field extent of intraarea interactions and may thus reduce their effect on perceptual size illusions.

\section{Concluding remarks}

These techniques extend pRF model-based analysis to probe underlying cortical architecture using fMRI. They also reveal the transformations that occur between different visual field maps. They show that in V1 pRF size, CMF and cortical surface area are related. V2, V3, and to some degree $\mathrm{hV} 4$, are still influenced by the V1 architecture as their cc-pRFs are constant. This underscores the importance of $\mathrm{V} 1$ architecture as a reference frame for subsequent processing stages and ultimately perception.

\section{References}

Albright TD, Desimone R (1987) Local precision of visuotopic organization in the middle temporal area (MT) of the macaque. Exp Brain Res 65:582-592.

Albus K (1975) A quantitative study of the projection area of the central and the paracentral visual field in area 17 of the cat. I. The precision of the topography. Exp Brain Res 24:159-179.

Amano K, Wandell BA, Dumoulin SO (2009) Visual field maps, population receptive field sizes, and visual field coverage in the human $\mathrm{MT}+\mathrm{com}-$ plex. J Neurophysiol 102:2704-2718.

Andrews TJ, Halpern SD, Purves D (1997) Correlated size variations in human visual cortex, lateral geniculate nucleus, and optic tract. J Neurosci 17:2859-2868.

Brainard DH (1997) The Psychophysics Toolbox. Spat Vis 10:433-436.

Brewer AA, Liu J, Wade AR, Wandell BA (2005) Visual field maps and stimulus selectivity in human ventral occipital cortex. Nat Neurosci 8:1102-1109.

Daniel PM, Whitteridge D (1961) The representation of the visual field on the cerebral cortex in monkeys. J Physiol 159:203-221.

DeYoe EA, Carman GJ, Bandettini P, Glickman S, Wieser J, Cox R, Miller D, Neitz J (1996) Mapping striate and extrastriate visual areas in human cerebral cortex. Proc Natl Acad Sci U S A 93:2382-2386.

Dougherty RF, Koch VM, Brewer AA, Fischer B, Modersitzki J, Wandell BA (2003) Visual field representations and locations of visual areas V1/2/3 in human visual cortex. J Vis 3:586-598.

Dow BM, Snyder AZ, Vautin RG, Bauer R (1981) Magnification factor and receptive field size in foveal striate cortex of the monkey. Exp Brain Res 44:213-228.

Dumoulin SO, Wandell BA (2008) Population receptive field estimates in human visual cortex. Neuroimage 39:647-660.

Dumoulin SO, Hoge RD, Baker CL Jr, Hess RF, Achtman RL, Evans AC (2003) Automatic volumetric segmentation of human visual retinotopic cortex. Neuroimage 18:576-587.

Duncan RO, Boynton GM (2003) Cortical magnification within human primary visual cortex correlates with acuity thresholds. Neuron 38:659-671.

Ejima Y, Takahashi S, Yamamoto H, Fukunaga M, Tanaka C, Ebisu T, Umeda $M$ (2003) Interindividual and interspecies variations of the extrastriate visual cortex. Neuroreport 14:1579-1583.

Engel SA, Rumelhart DE, Wandell BA, Lee AT, Glover GH, Chichilnisky EJ, Shadlen MN (1994) fMRI of human visual cortex. Nature 369:525.

Fiorani M Jr, Gattass R, Rosa MG, Sousa AP (1989) Visual area MT in the Cebus monkey: location, visuotopic organization, and variability. J Comp Neurol 287:98-118.

Friston KJ, Fletcher P, Josephs O, Holmes A, Rugg MD, Turner R (1998) Event-related fMRI: characterizing differential responses. Neuroimage 7:30-40.

Gattass R, Gross CG (1981) Visual topography of striate projection zone (MT) in posterior superior temporal sulcus of the macaque. J Neurophysiol 46:621-638.

Glover GH (1999) Deconvolution of impulse response in event-related BOLD fMRI. Neuroimage 9:416-429.

Hetherington PA, Swindale NV (1999) Receptive field and orientation scatter studied by tetrode recordings in cat area 17. Vis Neurosci 16:637-652.

Hubel DH, Wiesel TN (1962) Receptive fields, binocular interaction and functional architecture in the cat's visual cortex. J Physiol 160:106-154.

Hubel DH, Wiesel TN (1974) Uniformity of monkey striate cortex: a parallel relationship between field size, scatter, and magnification factor. J Comp Neurol 158:295-305.

Hubel DH, Wiesel TN (1977) Ferrier lecture. Functional architecture of macaque monkey visual cortex. Proc R Soc Lond B Biol Sci 198:1-59. 
Kay KN, Naselaris T, Prenger RJ, Gallant JL (2008) Identifying natural images from human brain activity. Nature 452:352-355.

Larsson J, Heeger DJ (2006) Two retinotopic visual areas in human lateral occipital cortex. J Neurosci 26:13128-13142.

le Gros Clark WE (1941) The laminar organization and cell content of the lateral geniculate body in the monkey. J Anat 75:419-433.

Levin N, Dumoulin SO, Winawer J, Dougherty RF, Wandell BA (2010) Cortical maps and white matter tracts following long period of visual deprivation and retinal image restoration. Neuron 65:21-31.

McIlwain JT (1986) Point images in the visual system—new interest in an old idea. Trends Neurosci 9:354-358.

Motter BC (2009) Central V4 receptive fields are scaled by the V1 cortical magnification and correspond to a constant-sized sampling of the V1 surface. J Neurosci 29:5749-5757.

Nestares O, Heeger DJ (2000) Robust multiresolution alignment of MRI brain volumes. Magn Reson Med 43:705-715.

Olman CA, Inati S, Heeger DJ (2007) The effect of large veins on spatial localization with GE BOLD at $3 \mathrm{~T}$ : displacement, not blurring. Neuroimage 34:1126-1135.

Pelli DG (1997) The VideoToolbox software for visual psychophysics: transforming numbers into movies. Spat Vis 10:437-442.

Perry VH, Cowey A (1985) The ganglion cell and cone distributions in the monkey's retina: implications for central magnification factors. Vision Res 25:1795-1810.

Pitman EJG (1939) A note on normal correlation. Biometrika 31:9-12.

Rosa MG, Sousa AP, Gattass R (1988) Representation of the visual field in the second visual area in the Cebus monkey. J Comp Neurol 275:326-345.

Schira MM, Tyler CW, Breakspear M, Spehar B (2009) The foveal confluence in human visual cortex. J Neurosci 29:9050-9058.

Schira MM, Tyler CW, Spehar B, Breakspear M (2010) Modeling magnification and anisotropy in the primate foveal confluence. PLoS Comput Biol 6:e1000651.
Schwarzkopf DS, Song C, Rees G (2011) The surface area of human V1 predicts the subjective experience of object size. Nat Neurosci 14:28-30.

Sereno MI, Dale AM, Reppas JB, Kwong KK, Belliveau JW, Brady TJ, Rosen BR, Tootell RB (1995) Borders of multiple visual areas in humans revealed by functional magnetic resonance imaging. Science 268:889-893.

Smith AT, Singh KD, Williams AL, Greenlee MW (2001) Estimating receptive field size from fMRI data in human striate and extrastriate visual cortex. Cereb Cortex 11:1182-1190.

Smith SM, Jenkinson M, Woolrich MW, Beckmann CF, Behrens TE, Johansen-Berg H, Bannister PR, De Luca M, Drobnjak I, Flitney DE, Niazy RK, Saunders J, Vickers J, Zhang Y, De Stefano N, Brady JM, Matthews PM (2004) Advances in functional and structural MR image analysis and implementation as FSL. Neuroimage 23 [Suppl 1]:S208-S219.

Stensaas SS, Eddington DK, Dobelle WH (1974) The topography and variability of the primary visual cortex in man. J Neurosurg 40:747-755.

Teo PC, Sapiro G, Wandell BA (1997) Creating connected representations of cortical gray matter for functional MRI visualization. IEEE Trans Med Imaging 16:852-863.

Van Essen DC, Newsome WT, Maunsell JH (1984) The visual field representation in striate cortex of the macaque monkey: asymmetries, anisotropies, and individual variability. Vision Res 24:429-448.

Wandell BA, Chial S, Backus BT (2000) Visualization and measurement of the cortical surface. J Cogn Neurosci 12:739-752.

Wandell BA, Dumoulin SO, Brewer AA (2007) Visual field maps in human cortex. Neuron 56:366-383.

Winawer J, Horiguchi H, Sayres RA, Amano K, Wandell BA (2010) Mapping hV4 and ventral occipital cortex: the venous eclipse. J Vis 10:1.

Worsley KJ, Liao CH, Aston J, Petre V, Duncan GH, Morales F, Evans AC (2002) A general statistical analysis for fMRI data. Neuroimage 15:1-15.

Zuiderbaan W, Ress D, Harvey BM, Green CA, Dumoulin SO (2010) Modeling center-surround configurations in population receptive fields using fMRI. Soc Neurosci Abstr 36:325.10. 